\title{
Origins of metastasis in breast cancer
}

\author{
By Kai-Jye Lou, Staff Writer
}

Researchers at the Albert Einstein College of Medicine of Yeshiva University and the Centocor Ortho Biotech Inc. unit of Johnson \& Johnson have linked multiple prometastatic processes in breast cancer to the production of the chemokine CCL2 in tumor cells. ${ }^{1}$ The findings could aid the development of new therapeutics to prevent breast cancer metastasis, the main cause of breast cancer mortality in Western women, ${ }^{2}$ and could point to a new indication for the company's CNTO 888.

CNTO 888, an antibody against human monocyte chemoattractant protein-1 (MCP-1; CCL2), is in Phase II testing to treat metastatic, castration-resistant prostate cancer and Phase I trials for solid tumors. The compound also is in Phase II testing to treat idiopathic pulmonary fibrosis. ${ }^{3}$

CCL2 signals through CC chemokine receptor 2 (CCR2; CD192) and has been identified as a therapeutic target in breast cancer. ${ }^{4,5}$ Signaling through this chemokine-receptor pair has been associated with multiple processes that promote tumor progression and metastasis, like macrophage recruitment into the tumor microenvironment.

"Most treatments for breast cancer work by targeting the tumor cells themselves, but the CCL2-targeting approach would uniquely affect an interaction that these cells have with the tumor microenvironment." - Leisha Emens, The Sidney Kimmel Comprehensive Cancer Center at The Johns Hopkins University

breast tumor cells disseminating from the primary malignancy, recruits CCR2 $2^{+}$inflammatory monocytes. These monocytes in turn recruit and differentiate into metastasis-associated macrophages. Finally, the macrophages promote tumor cell extravasation and metastatic seeding in a VEGF-A-dependent manner.

In multiple mouse models of human breast cancer, both CNTO 888 and a $\mathrm{mAb}$ targeting $\mathrm{Ccl} 2$ significantly decreased the frequency of pulmonary metastasis and increased survival compared with saline
( $p<0.001$ for both). The anti-CCL2 mAbs also blocked tumor cell migration in vitro compared with a control antibody $(p<0.001$ for anti-Ccl2 $\mathrm{mAb} ; p<0.01$ for CNTO 888).

Conditional ablation of Vegf- $a$ in inflammatory monocytes or bone marrow-derived macrophages inhibited the metastatic seeding of mouse mammary cancer cells in the lung. That finding suggests that combining a CCL2 inhibitor with a VEGF-A inhibitor could have an even stronger antimetastatic effect than either compound alone.

Results were published in Nature.

\section{Preventing migration}

Jeffrey Pollard, corresponding author on the paper and deputy director of the Albert Einstein Cancer Center at the university, said the study suggests that inhibitors of monocyte recruitment and function could have a significant impact on prometastatic processes in breast cancer.

"Such a therapy can be realized, for example, through anti-CCL2 antibodies and antimacrophage treatments," he told SciBX. "Our work also demonstrates a dynamic interaction between tumor cells and nontumor cells that promotes metastasis."

"What this study suggests is that rather than deplete the macrophages directly, what one might want to do is prevent these cells from migrating to the tumor and sites of metastasis," added Amy Fulton, a professor in the Department of Pathology at the University of Maryland School of Medicine and the University of Maryland Greenebaum Cancer Center. "The theory is interesting and sound based on the known biology of these cells. CCL2 is one of the normal signaling molecules used by macrophages and other cells to migrate to various parts of the body."

In addition, Pollard noted that although CCL2 had a mosaic-like expression pattern in the primary tumor, the chemokine was expressed throughout metastatic lesions. "This finding suggests that CCL2-expressing tumor cells may be the subpopulation that preferentially extravasate from the primary tumor and migrate to metastatic sites," he said.

If correct, this could help identify patients at increased risk for developing metastatic disease who would be candidates for an anti-CCL2 therapy.

"From our experimental data, we believe that extravasation is one of the key rate-limiting steps for the development of metastatic lesions in breast cancer," Pollard told SciBX.

The consensus among researchers contacted by $S c i B X$ is that a CCL2-targeting therapy would most likely be evaluated in the clinic as part of combination therapy.

"There are already many different treatments for breast cancer, so it will be important to determine how a CCL2-targeting compound could be integrated with such treatments and whether there are synergistic or antagonistic interactions between them," said Leisha Emens, an associate professor of oncology at The Sidney Kimmel Comprehensive Cancer Center at The Johns Hopkins University. "Most treatments for breast cancer work by targeting the tumor cells themselves, but the CCL2-targeting approach would uniquely affect an interaction that these cells have with the tumor microenvironment." 


\section{ANALYSIS}

"What one might want to consider is combining an anti-CCL2 or antimacrophage therapy with chemotherapy to see if it results in an enhancement of therapeutic outcomes" such as improving survival, said Pollard.

Kristiina Vuori, president of the Sanford-Burnham Medical Research Institute and director of its cancer center, added that another logical duo would be a CCL2-targeting therapy and an anti-VEGF drug.

"Most of the experiments performed in the current study were designed to test the hypothesis that blocking CCL2 can inhibit early processes in breast cancer metastasis, but this may not be representative of its use in the clinical setting, where cancer cell dissemination may have already occurred," Vuori added. "They will need to determine the timing of the effect of the CCL2-targeting antibody. It will be important to determine whether such a therapy would be effective after metastasis has been established in distant sites."

Fulton noted that chemokine receptors can be activated by multiple ligands and that breast cancer cells themselves express multiple chemokine receptors linked to prometastatic processes.

Because of this high degree of redundancy in the chemokine system, "I think it's good to be skeptical on whether blocking any single target in the chemokine system will have a long-term therapeutic effect," said Fulton. "While such treatments may show an initial therapeutic effect, the cancer may quickly develop an escape mechanism. It will be important to carry out longer-term studies to determine if the breast cancer will start using another chemokine receptor or ligand to spread."

Indeed, a 2003 study has shown that MCP-2 (CCL8) and MCP-3 (CCL7) also are ligands for CCR $2 .^{6}$

Pollard said his group is focused on elucidating additional details of the metastatic cascade in breast cancer. Although the current study looked primarily at breast cancer that metastasized to the lung, Pollard said the group is studying whether other common sites of metastasis in breast cancer use the CCL2-mediated pathway.

According to Pollard, his group has not filed for a patent on the findings described in the paper.

In 2008, J\&J filed a patent application covering anti-CCL2 antibodies and their uses in multiple indications including cancer. The company did not disclose whether it plans to develop CNTO 888 for metastatic breast cancer.

Lou, K.-J. SciBX 4(25); doi:10.1038/scibx.2011.699

Published online June 23, 2011

\section{REFERENCES}

1. Qian, B.-Z. et al. Nature; published online June 8, 2011; doi:10.1038/nature10138

Contact: Jeffrey W. Pollard, Albert Einstein College of Medicine of Yeshiva University, New York, N.Y. e-mail: pollard@aecom.yu.edu

2. Weigelt, B. et al. Nat. Rev. Cancer 5, 591-602 (2005)

3. Schaeffer, S. BioCentury 19(26), A13-A15; June 20, 2011

4. Soria, G. \& Ben-Baruch, A. Cancer Lett. 267, 271-285 (2008)

5. Lu, X. \& Kang, Y. J. Biol. Chem. 284, 29087-29096 (2009)

6. Vande Broek, I. et al. Br. J. Cancer 88, 855-862 (2003)

\section{COMPANIES AND INSTITUTIONS MENTIONED} Albert Einstein College of Medicine of Yeshiva University, New York, N.Y.

Johnson \& Johnson (NYSE:JNJ), New Brunswick, N.J. Sanford-Burnham Medical Research Institute, La Jolla, Calif. The Sidney Kimmel Comprehensive Cancer Center at The Johns Hopkins University, Baltimore, Md. University of Maryland Greenebaum Cancer Center, Baltimore, Md. University of Maryland School of Medicine, Baltimore, Md. 